\title{
Sacrococcygian Teratoma in a Newborn: Four Years After the Surgery-A Case Report
}

\author{
S Messaoudi*, H Guerouaou, FZ Smaili, A Ayyad, A Ammor, D Azzouzi and R Amrani \\ Maternal and Child Health Laboratory, University Mohammed first of Oujda, Morocco \\ *Corresponding author: S Messaoudi, Maternal and Child Health Laboratory, faculty of medicine and pharmacy of Oujda, universi- \\ ty Mohammed first of Oujda, Morocco.
}

To Cite This Article: S Messaoudi, H Guerouaou, FZ Smaili, A Ayyad, A Ammor, D Azzouzi, R Amrani, Sacrococcygian Teratoma in a Newborn: Four Years After the Surgery-A Case Report. Am J Biomed Sci \& Res. 2021 - 12(2). AJBSR.MS.ID.001734. DOI: 10.34297/AJBSR.2021.12.001734.

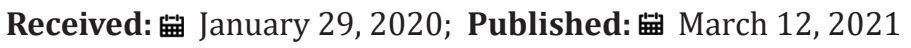

\begin{abstract}
Sacrococcygeal teratoma is a rare congenital tumor, occurs one newborn in 35,000 to 40,000 live births. Although it's a rarity, it is the most common tumor of the fetus and the neonate. These teratomas are derived from all three layers. Mostly benign, sacrococcygeal teratomas are four times more common in females than males. The prognosis of this disease is closely related to early diagnosis, which is now possible in antenatal (usually made between 22 weeks of gestation) through obstetric ultrasound, enabling better management peri-natal and post these children. Postnatal diagnosis is based on clinical, ultrasound and MRI, which allows classifying the tumor according to Altman's classification and eliminates the main differential diagnosis: meningocele.
\end{abstract}

We report the case of a sacrococcygian teratoma discovered at birth in a female neonate, born from a well-attended pregnancy, in which the diagnosis could have been evoked in antenatal. After the various necessary additional examinations, the patient was taken care of at day 15 by the pediatric surgery team. Radical resection must be performed to avoid potentially malignant recurrence even if the primary lesion is benign.

Keywords: Sacrococcygian Teratoma; MRI; Early surgery; Complications

\section{Summarize}

Sacrococcygian teratoma is a rare congenital tumor $(1$ in 35,000-40,000), derived from the three embryonic leaves. Despite its rarity, it represents the most common tumour in the neonatal period. This tumor is most often benign, which occurs most frequently in females with one with a sex ratio of $1 / 4$. Its prognosis is closely related to the early diagnosis, which is now possible in antenatal (usually between 22 and 34 SA) thanks to obstetric ultrasound. Post-natal diagnosis is based on clinical, ultrasound and spinal cord MRI, which classifies the tumour according to the Altman classification and eliminates the main differential diagnosis: meningocele. Tumor excision must be radical to prevent recurrence which is readily done in malignant form. We report the case of a sacrococcygian teratoma discovered at birth in a female newborn, from a well-followed pregnancy, in which the diagnosis could have been evoked antenatal. After carrying out the necessary additional examinations, the patient was treated at J15 by the pediatric surgery team.

\section{Clinical Case}

This is a female newborn born to a non-inbred parent from a well-attended pregnancy (7 ultrasounds) conducted at 34 SA

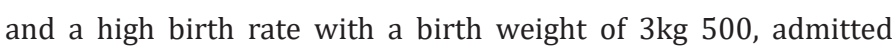
to our department on the five day of life for taking charge of a mass of the gluteus region. In addition, the family reported no significant history of disease. The admission examination found a hemodynamic and respiratory stable newborn; with an irregular surface polylobed cystic mass in the buttock area with a repressed anal orifice forward (Figure 1). The rest of the clinical examination was without particularity. As part of the etiological investigation a series of additional examinations was carried out: 

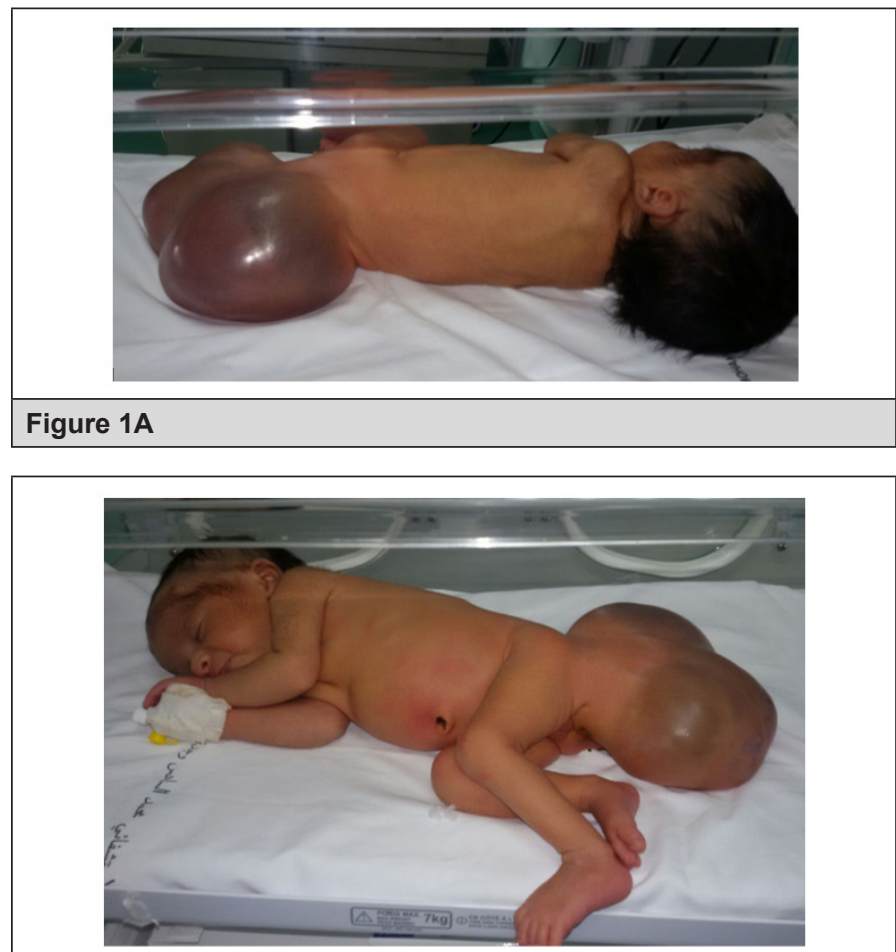

Figure 1B

Figure 1 (A-B): Sacrococcygian Teratoma A: Anterior View/B: Posterior

Standard radiography: shows the presence of voluminous opacity in the sacrococcygian region, fluid tone, or absence of calcifications (Figure 2).

Ultrasound mass: Multiloculate cystic mass measuring approximately $23 * 18 \mathrm{~cm}$ may be in favor of a teratoma, a hemangioma cannot be eliminated.

Abdominal-pelvic ultrasound: was without peculiarity.

The medullary MRI: A large cloisonné sacro coccygian cyst of type II (teratoma) attached in its internal part to the wall of the rectum without spinal cord injury.

Tumor markers: beta-human chorionic gonadotropin ( $\beta H C G$ ): $2.42 \mathrm{mUI} / \mathrm{ml}$; Alpha-Fetoprotein (AFP)>20,000 ng/ml.

The impact and preoperative balance: $\mathrm{NFS}: \mathrm{Hb}$ at $19,5 \mathrm{~g} /$ dl; GB at 10,000 /mm3; Plq at 325,000/mm3; CRP: $0.6 \mathrm{mg} / \mathrm{l}$; the ionogram and the crash balance came back normal.

The malformative balance: Transfontanal ultrasound returning normal; transthoracic ultrasound objectified a CIA at 5 mm without impact.

After the condition, the newborn received a mass resection (Figures 3-5) with regular follow-up in pediatric consultation and pediatric surgery followed in pediatric consultation and pediatric surgery every 3 months. Apart from its aesthetic problem (Figures 6 \& 7), we noted a good evolution of the child both on the height, weight and psychomotor plane with a conservation of the sphincter function which was a satisfying result for both family and doctors. And then plastic surgeons take over to take care of the aesthetic damage.
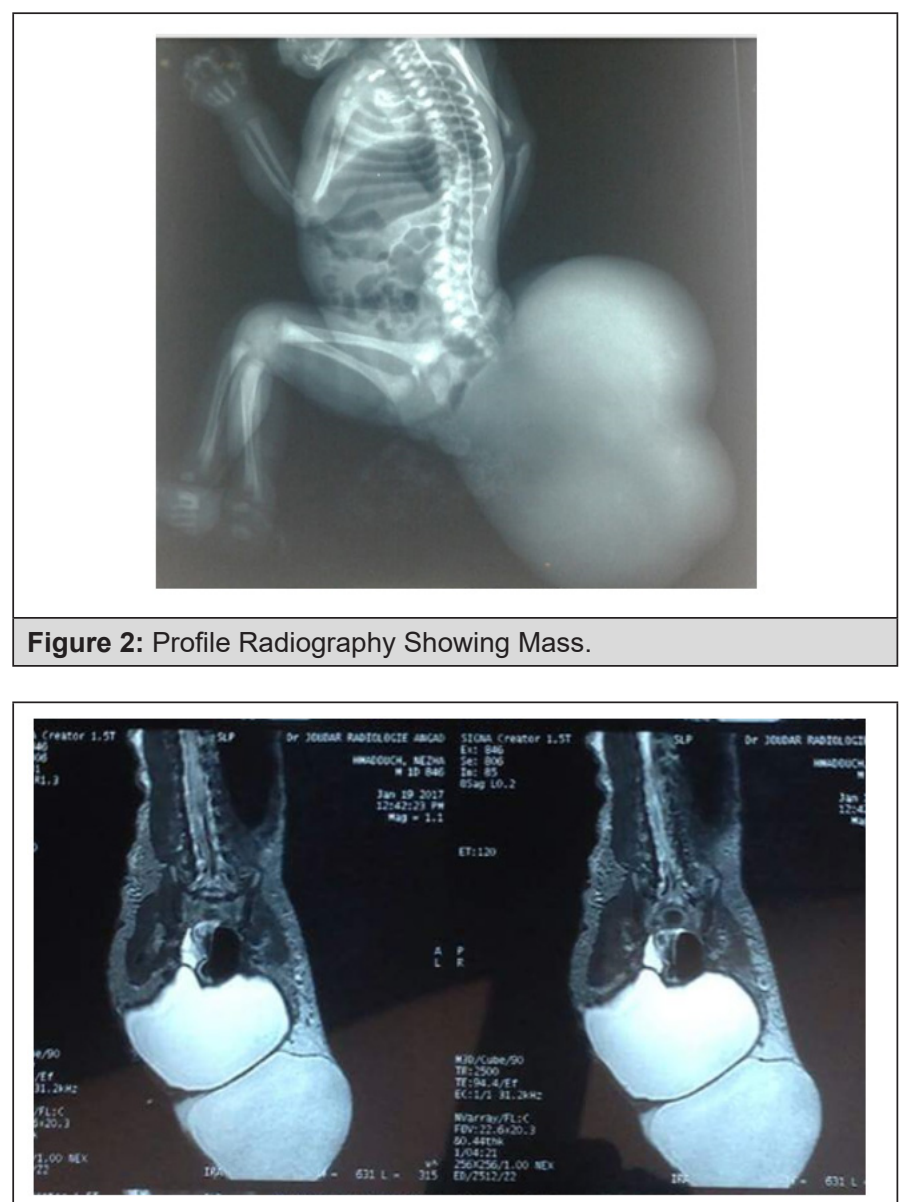

Figure 3A: Frontal Slices

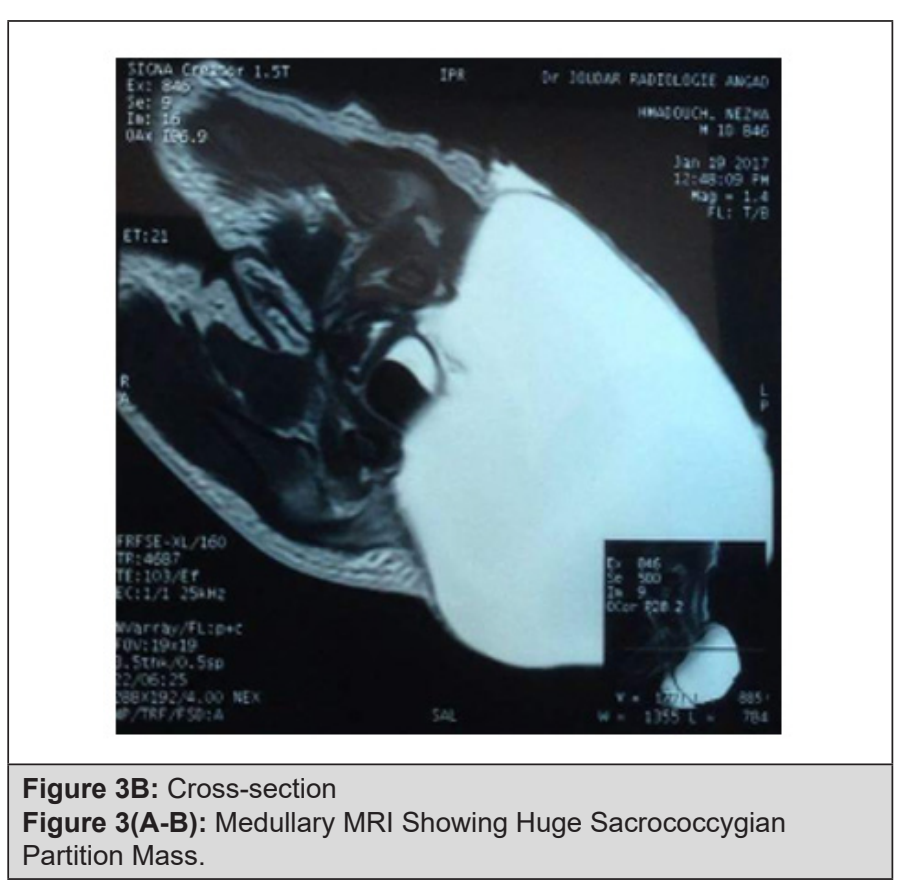



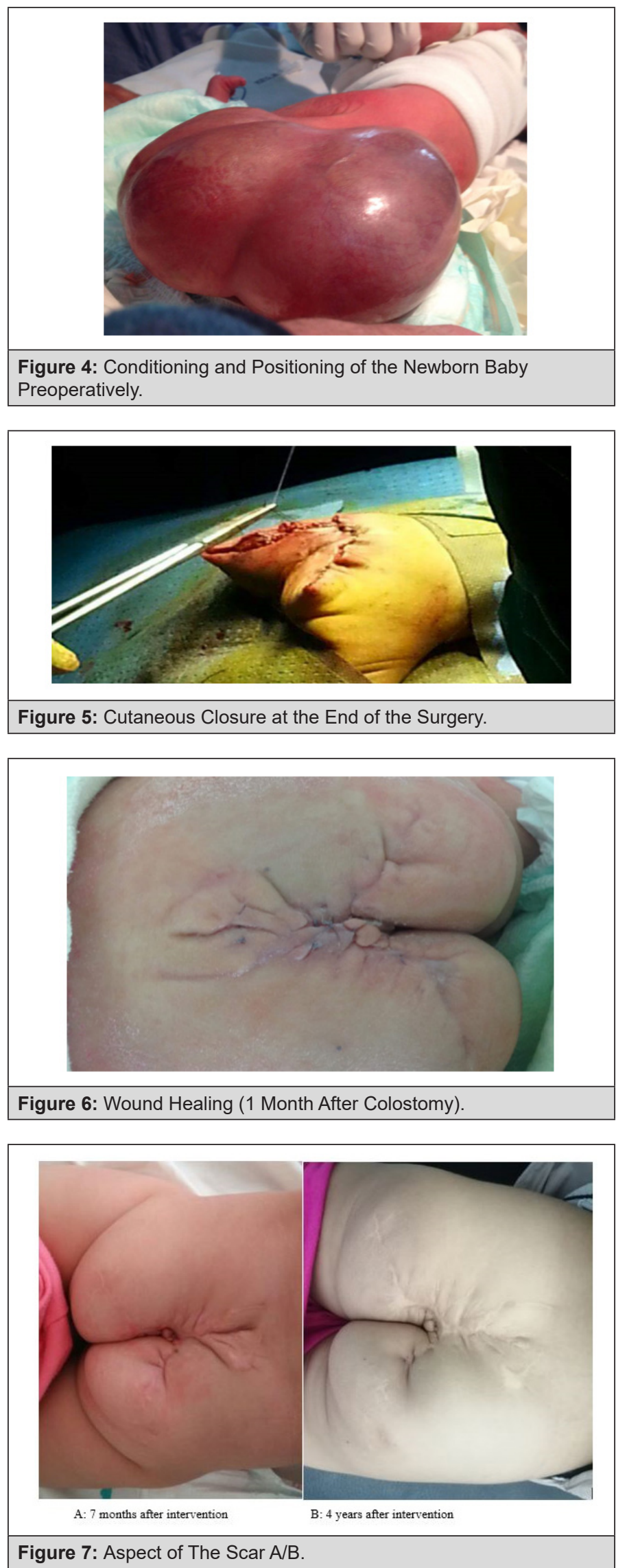

\section{Discussion}

TSC is a rare congenital tumor with an overall incidence variant between 1 in 35,000 and 40,000 live births [1,2], Despite this rarity the TSC occupies the first rank of teratomas of the new-born; the 2nd rank of neonatal tumors after lymphangiomas and the 3rd rank of retro-peritoneal tumors of the child, after neuroblastomas and nephoblastomas $[3,4]$. Sacro-coccygian localization is the first of the teratoma sites affecting small children, in a proportion varying from $40 \%$ [5] to about $60 \%$ [6]. Due to their early development in the womb, sacro-coccygian teratomas are present at birth. In the series of Altman et al. [1] more than half of the patients (225 cases) were diagnosed on the first day of life as in our case. Forms diagnosed at birth remain the most common. This was reported by several authors $[1,7,8]$. Some family cases of TSC have been described by Ashcraft \& Holder [9] who suggest that the tumor is inherited autosomally dominant. This family form has several characteristics that distinguish it from the usual TSC: The discovery of a sacral defect or an ano-rectal malformation must search for a pre-sacral mass. Similarly, the coexistence of an anal stenosis, a sacral defect and a presacral teratoma in a patient, must encourage screening in the family. A thorough examination and examination may reveal family members affected by the illness. Constipation is the main symptom of discovery. Twine is found as a family history in a significant number of patients with a rate of 30 to $50 \%[10,11]$ above that of the normal population.

The discovery of these tumors during pregnancy allows better management and improves the fetal and maternal prognosis. The first cases of antenatal diagnosis of TSC were described by HORGER and VERMA in 1979 [11]. Ultrasound plays an important role in the in-utero discovery of TSC, usually the diagnosis is made between 22 and $34 \mathrm{SA}$, but with the advent of two-dimensional ultrasound then three-dimensional one can currently evoke the diagnosis from the first trimester [12].

Most CHTs are diagnosed or visible at birth. This is likely due to the fact that the majority of CHTs are predominantly external (total 82\%) [13], but in other cases where the external component is absent, the mode of revelation varies according to its prolonged evolution and its locoregional extension which may concern all the structures adjacent to the teratoma. Magnetic Resonance Imaging (MRI) provides the baseline examination that characterizes the nature of the mass, and the links to the muscular and bony structures of the pelvis (upper strait). And to highlight an intrapelvic extension although the relations with the rectum are less visible than with the scanner. It is particularly effective for the study of the spine, so to visualize an intra-spinal extension and/or remove the doubt on meningocele which remains the first differential diagnosis of TSC.

Elevated serum AFP is the best indicator of endodermal sinus tumour [13-15] hence its indication and diagnostic value and 
prognosis as it is considered an important key for the clinical differentiation between benign and malignant teratomas, as well as for the evaluation of the effectiveness of treatment. Tsuchida and Hasegawa [13] conducted a study of 61 Teratomes cases in which they analyzed the relationship between AFP and histological type of tumour. Based on the results of this study, they concluded that the AFP rate is almost always high for malignant teratomas, but it is exceptionally high for mature teratomas. For immature teratomas, the number of cases studied was insufficient to conclude. The same result was obtained by Billmire \& Grosfeld [11]. The determination of alpha-foetoprotein and beta HCG is essential before starting the treatment and it is desirable for the same patient that this determination be carried out with the same kits throughout the treatment.

Histopathological examination is not necessary for secreting tumors because the diagnosis can be confirmed on the presence of high secretion of tumor markers malignant teratomas. On the contrary, it is essential for non-secreting tumors (biopsy of the tumor or superficial lymphadenopathy) and will involve immunohistochemical studies looking for alpha-foetoprotein and beta HCG secretion wherever possible [16-18]. Diagnosed antenatal HCT requires rigorous obstetric surveillance. However, the possible complications of hyper-vascularized forms of TSC make a more interventionist approach more permissible than simple prenatal surveillance $[19,20]$. This in utero therapeutic approach aims either to remove the tumor or to reduce its vascularization, thereby reducing its growth potential [21].

Obstetric management and choice of delivery route depend on several factors (Figure 8), and it must be programmed. The newborn must be immediately referred to the neonatologist with the fastest possible pediatric surgical management. Tumor excision is the only effective treatment for TSC. It is indicated as soon as the diagnosis is made, even in the first days of life, given the risk of cancer which is not negligible. According to Altman et al. [1] the percentage of malignancy when the diagnosis is made before two months, is $10 \%$ for boys and $7 \%$ for girls. While for patients diagnosed after two months, $2 / 3$ of boys (67\%) and almost half of girls (48\%) had malignant tumors. In addition, it appears that boys are more prone to malignant transformation of CHTs than girls. Selon NEUBERT [22] The rate of malignant tumors may increase in relation to the age of diagnosis of TSC: at birth $2 \%$ malignancy; at 2 months $10 \%$ malignancy; at 6 months $60 \%$ malignancy. Hence the interest of an early diagnosis and exeresis for a better prognosis.

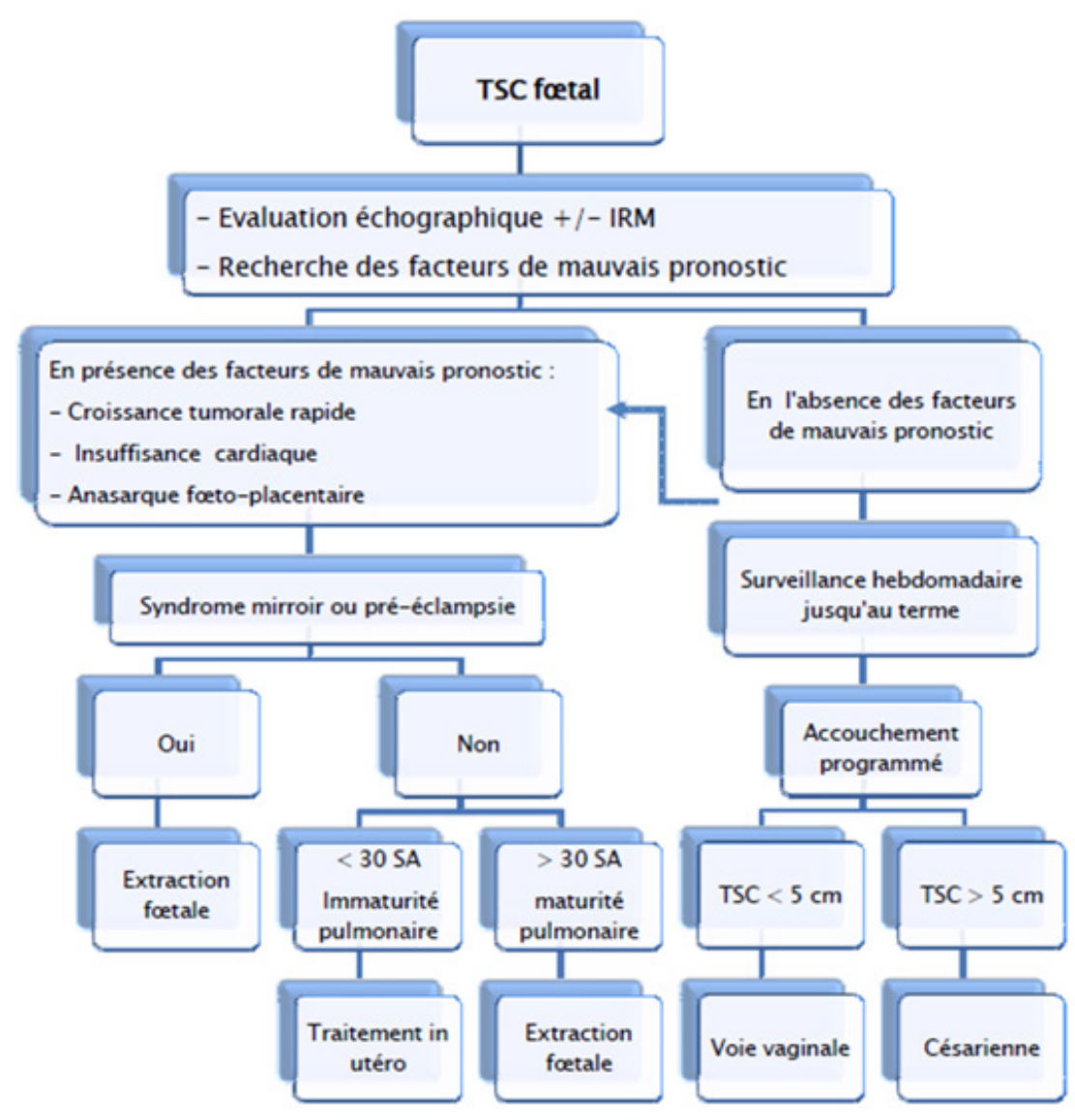

Figure 8: Fetal TSC Obstetric Management Algorithm [25]. 
Since the introduction of polychemotherapy in the treatment of malignant TSC, the prognosis has improved significantly. Its effectiveness is clearly demonstrated in the literature. Dewan et al. [18] reported a series of 57 patients with TSC, treated between 1948 and 1986. Prior to 1975, malignant teratomas were treated with surgery or radiotherapy, and in some patients with mono chemotherapy. But no patient survived. In 1976, intensive polychemotherapy was introduced, followed by surgical excision, with or without post-operative radiotherapy, 3 out of 5 patients survived and are almost cured. The same goes for Shanbhog et al. [23] they reported an average survival of two months for patients treated with surgery +/- radiotherapy, while patients who received chemotherapy had an average survival of 11 months. Therefore, radiotherapy is only indicated for post-chemotherapy residues that cannot be re-sequenced.

Follow-up of these patients is necessary after tumor resection in order to detect early recurrence. It must be clinical, biological and radiological. A complete clinical examination, including a rectal touch, should be performed regularly, looking for a tumor syndrome (palpable mass, signs of urinary or digestive compression). The rectal touch can be alternated with a pelvic ultrasound to avoid a possible psychological trauma induced by repetitive rectal touching. Biologically, the dosage of AFP is an excellent means for postoperative follow-up, since it falls after tumor resection, and re-ascendes in case of recurrence or metastasis. According to the study by Barreto et al. [15], The time required for normalization of AFP levels after tumor resection varies between 6 and 12 months ( 9 months on average), however, Bilik et al. [16] recommend a AFP dose every 3 to 6 months for at least 3 years after surgery. The hCG is another tumor marker whose increase testifies the presence of choriocarcinoma, and which can be used in post-operative followup generally normalizes in 8 to 50 days if the tumor excision is complete. A confirmed secondary elevation of the AFP or hCG rate indicates recurrence or metastasis, Sometimes previous several months any clinical signs. The diagnosis of recurrence must also perform a radiological assessment including a chest x-ray for lung metastases, an ultrasound and especially a CT scan that detects subclinical tumor recurrence, assess the relationship of tumour recurrence with adjacent structures and look for retroperitoneal lymphadenopathy. The MRI mainly allows to look for intraspinal invasion. This check-up must be carried out every 3 months for the 2 years following the surgery, then every 6 months for 2 more years. However, reported cases of recurrence in adulthood lead to failure to permanently release these patients even after this delay [24,25].

The study concerning our case allows us to evoke the problem of complications related to management; the hemorrhagic shock was the most redoupted complication, especially with such a tumor that is still very voluminous and hyper vascularized. The newborn received a transfusion of 4 globular units during the procedure. In the series of Altman et al. [1] 9 out of 63 deaths are due to intraoperative bleeding. Murphy et al. [26] report two deaths secondary to massive hemorrhage, one died during surgery. The other, who ruptured the tumour during childbirth, died before the surgery.

In post-operative procedures, all measures have been taken to avoid complications related to the wound $[27,28]$ : scars away from the anus, drain during surgery, maintaining ventral decubitus, broad-spectrum antibiotic therapy with rigorous asepsis. Yet the wound got infected with secondary sutures. Faced with this complication, the realization of a colostomy seemed to be necessary, and the newborn was taken back to the block with an interval of 10 days between the two surgical gestures. The evolution was favorable, marked by the healing of the wound.

Based on this history and in parallel with the literature, it can be concluded that the infectious risk in this type of intervention is major, and colostomy remains one of the useful alternatives that has shown its effectiveness in managing this complication. Functional complications were also reported in the literature [29], with constipation being the most common. Fortunately, the functional result in our patient was better without urinary or rectal incontinence. The aesthetic damage that remains one of the most common complications of this type of procedure will be dealt with later by the plastic surgeons. Nevertheless, the emotional and psychological impact of this kind of procedure is very common and mostly neglected by practitioners in favor of the functional results. In our case, the child at the age of four has begun noticing her sacral scar which is a source of emotional destress needing a specialist assistance.

\section{Conclusion}

Sacrococcygian teratoma is a rare tumour although it is the most common neonatal tumour. Neonatal and postnatal management are currently well codified and have been significantly improved by the constant and still recent appearance of ultrasound techniques. Treatment at birth, even in the first days of life, is surgical. The prognosis of TSC is generally good. Indeed, in benign teratomas, the early and total surgery carrying the coccyx gives good results. The prognosis of malignant teratomas has improved significantly thanks to chemotherapy. The surveillance of these children will be strengthened during the first year (rectal touch, ultrasound, alphafoetproteine) and attentive during the following three years, because of the risk of secondary degeneration, but also the rate of significant aesthetic and functional sequelae.

\section{Acknowledgement}

None. 


\section{Conflict of Interest Statement}

No conflict of interest.

\section{References}

1. Altman RP, Randolph JG, Lilly JR (1974) Sacrococcygeal Teratoma: American Academy of Pediatrics Surgical Section Survey. J Pediatr Surg 9(3): 389-398.

2. Pantoja E, Lopez E (1978) Sacrococcygeal teratomas in infancy and childhood. J Med 78: 8136

3. Benamar A, Ben Abdellah F, Ben Hammou M (1998) Les tératomes sacro-coccygiens: à propos de 21 cas. Clinique chirurgicale C Hôpital des enfants. CHU Rabat. Maroc Médical, Tome 20 N², 117-120.

4. Schauvliege J, Gondry Jouet C, Gondry J (2009) RADIOANATOMIE FOETALE EN IRM. Service de radiologie A, CHU AMIENS.

5. Izant RJ, Filston HC (1975) Sacrococcygeal Teratoma Analysis of fortythree cases. Am J Surg 130(5): 617-621.

6. Tapper D, Lack EE (1983) Teratomas in infancy and childhood: A 54-Year Experience at the Children's Hospital Medical Center. Ann Surg 198(3): 398-410.

7. Niramis R, Anuntkosol M, Buranakitjaroen V, Tongsin A, Mahatharado $\mathrm{V}$, et al. Long-Term Outcomes of Sacrococcygeal Germ Cell Tumors in Infancy and Childhood. Surg Res Pract 2015: 398549.

8. Rescorla Fj, Sawin Rs, Coran Ag, Dillon Pw, Azizkhan Rg (1998) Longterm outcome for infants and children with sacrococcygeal teratoma: a report from the Childrens Cancer Group. J Pediatr Surg 33(2): 171-176.

9. Ashcraft Bw, Holder Tm (1974) Hereditary Presacral Teratoma. J Pediatr Surg 9(5): 691-697.

10. Gross SJ, Clatworthy HW, Mecker IA (1951) Sacrococcygeal Teratoma in infant and children. Surg Gynecol Ostet 92: 341-347.

11. Grosfeld JI, Ballanrine TV, Lowe D (1976) Benign and malignant sacrococcygeal teratomas in children: Analysis of 85 patients. Surgery 80(3): 297-305.

12. Wells Rg, Sty Jr (1990) Imaging of sacrococcygeal germ cell tumors. Radiographics 10(4): 701-713.

13. Mekki M, Jallouli M, Krichene I, M Belghith, Nouri A, et al. (2008) Les tératomes sacro-coccygiens. Experience d'un service de chirurgie pédiatrique nord-africain. Oncologie 9: 864-868.

14. Tsuchida Y, Hasegawa H (1983) The diagnostic value of alpha-fetoprotein in infantsand children with teratomas: a questionnaire survey in Japan. J Pediatr Surg 18(2): 152-155.
15. Barreto MW, Silva LV, Barini R, Oliveira Filho AG, Sbragia L (2006) Alphafetoprotein following neonatal resection of sacrococcygeal teratoma. Pediatr Hematol Oncol 23(4): 287-291.

16. Bilik R, Shandling B, Pope M, P Thorner, Ein SH, et al. (1993) Malignant benign neonatal sacrococcygeal teratoma. J pediatr Surg 28: 1158-1160.

17. Ouattara O, Dieth D (1997) Tératome sacro-coccygien: Essai de chimiothérapie Médecine d'Afrique Noire 44(3).

18. Dewan PA, Davidson PM, Campbell PE, Tiedemann K, Jones PG (1987) Sacrococcygeal teratoma: has chemotherapy improved survival? J Pediatr Surg 22(3): 274-277.

19. Gabra HO, Edwin C (2006) Sacrococcygeal teratoma-a 25-year experience in a UK regional center. Journal of Pediatric Surgery 41(9): 1513-1516.

20. Hunt Pt, Davidson Kc, Ashcraft K (1977) Radiography of hereditary presacral teratoma. Radiology 122(1): 187-190.

21. Langer JC, Harrison MR, Schmidt KG, Silverman NH, Anderson RL, et al. (1989) Fetal hydrops and death from sacrococcygeal teratoma: rationale for fetal surgery. Am J Obstet Gynecol 160(5 Pt 1): 1145-1150.

22. Neubert S, Trautmann K, Tanner B, Steiner E, Linke F, et al. (2004) Sonographic prognostic factors in prenatal diagnosis of Sacrococcygeal Teratoma. Fetal diagnosis and therapy19: 319-326.

23. Shanbhogue LKR, Gough DCS, Jones PM (1989) Malignant sacrococcygeal teratoma: improved survival with chemotherapy. Pediatr Surg Int 4: 202-204.

24. Huddart SN, Mann JR, Robinson K, Raafat F, Imeson J, et al. (2003) Children's Cancer Study Group.Sacrococcygeal teratomas: the UK Children's Cancer Study Group's experience. I Neonatal Pediatr Surg Int 19(1-2): 47-51.

25. Gross SJ, Benzie RJ, Sermer M, Skidmore MB, Wilson SR. (1987) Sacrococcygeal teratoma: prenatal diagnosis and management. Am J Obstet Gynecol 156(2): 393-396.

26. Murphy JJ, Blair GK, Fraser GC (1992) Coagulopathy associated with large sacrococcygeal teratomas. J Pediatr Surg 27(10): 1308-1310.

27. Gross Rw, Clatwrty Jr Hw, Meeker Jr Ia (1951) Sacrococcygeal teratomas in infants and children; a report of 40 cases. Surg Gynecol Obstet 92(3): 341-354.

28. Ein SH, Adeyemi SD (1980) Benign sacrococcygeal teratomas in infants and children: a 25-year review. Ann Surg 191(3): 382-384.

29. Draper H, Chitayat D, Ein SH, Langer JC (2009) Long-term functional results following resection of neonatal sacrococcygeal teratoma. Pediatr Surg Int. 25(3): 243-246. 